$$
\text { X }
$$

COMETARY DUST 


\title{
Composition and Optical Properties of Cometary Dust
}

\author{
Martha S. Hanner \\ Jet Propulsion Laboratory, California Institute of Technology, \\ Pasadena California 91109
}

\begin{abstract}
Comet dust consists primarily of silicate and carbonaceous material. The carbonaceous material is not yet well-characterized. The $3.36 \mu \mathrm{m}$ emission feature arises primarily from gas phase molecules, while a small feature at 3.29 $\mu \mathrm{m}$ may be due to aromatic hydrocarbons in the grains. Olivine, a high temperature condensate, is present in some comets. Polarization and albedo maps have shown that the coma is not uniform; an inner halo of large particles may be present.
\end{abstract}

\section{Introduction}

Comets formed in the outer solar nebula where the temperature remained low enough that interstellar grains could have been incorporated with little alteration. Studies of the solid grains release from comets can help us to understand the conditions in the solar nebula and the extent to which interstellar material was preserved in comets.

Comets apparently originate from two reservoirs in the outer solar system. The comets that populate the inner and outer Oort Cloud probably formed in the Saturn Neptune region (10 - 30 AU) and were dynamically scattered to the Oort Cloud. This is the source of new and long-period comets. Short-period comets, with their low inclination orbits, most likely originated in the trans-Neptune region at $30-100 \mathrm{AU}$ (Duncan et al. 1988). The largest objects in this population have now been directly detected (Jewitt \& Luu 1995).

If there were compositional gradients in the solar nebula, due to the gradient in temperature or to the extent of mixing between the warm inner regions of the nebula and the comet-forming regions, then one would expect to see differences between the long and short period comets and even within the two populations. Thus, studies of the heterogeneity among comets may lead to a better understanding of how the conditions varied in the solar nebula and the important question of radial transport of material.

No doubt, there are cometary particles among the IDPs collected at Earth and these represent an important resource for studying the processing history of the dust. The anhydrous chondritic aggregate IDPs are the most likely candidates, based on their structure and inferred atmospheric heating (Bradley 1995). However, it is important to verify this identification via independent observations of cometary dust.

The first in situ sampling of comet dust composition was obtained by the dust impact analyzer on the Halley probes (Kissel et al. 1986). A dust component rich in organic refractory material was discovered, the $\mathrm{CHON}$ particles. Usually, $\mathrm{C}, \mathrm{Si}$, and $\mathrm{Mg}$ occurred together, indicating that the silicate and carbonaceous material were well mixed 
on a submicron scale (Jessberger et al. 1988). These discoveries provide a framework for interpreting the remote sensing observations. However, the total sample of cometary dust analyzed by these instruments was less than one $10 \mu \mathrm{m}$ IDP.

In the next decade, the Rosetta mission will visit a comet and the STARDUST mission will return a sample of cometary dust for laboratory analysis. Yet remote sensing remains our best means of surveying the nature of the dust in many comets and comparing with the dust in the interstellar medium. This paper reviews the current status of our knowledge about cometary dust from remote sensing.

\section{Thermal Emission}

The 3.5 - $20 \mu \mathrm{m}$ spectral energy distribution of the thermal emission from the dust coma gives color temperatures that are $5-25 \%$ hotter than that of a theoretical blackbody. Color temperature is determined by the physical temperature of the grains and their wavelength-dependent emissivity. The temperature of a particle in the solar radiation field depends on the balance between the solar energy absorbed at visual wavelengths and the energy radiated in the infrared. Small silicate grains, which are transparent at visual wavelengths, absorb little solar energy yet radiate efficiently at $10 \mu \mathrm{m}$. Consequently, they will be cold. Small carbonaceous grains, on the other hand, absorb strongly at visual wavelengths, but cannot radiate efficiently in the infrared at wavelengths greater than about 10 times their size. Thus, they heat up until the energy radiated at $3-8 \mu \mathrm{m}$ balances the absorbed energy. In fact, for small absorbing grains, their size controls the temperature, regardless of the specific composition (Hanner 1983). Grains larger than a few microns will be hotter than a blackbody only if they are very fluffy (e.g. Xing \& Hanner 1995).

Thus, we can conclude, independent of a specific model, that the 3-20 $\mu \mathrm{m}$ thermal emission in most active comets arises predominantly from sub- $\mu \mathrm{m}$ to $\mu \mathrm{m}$ sized absorbing grains. Model calculations of the thermal spectral energy distribution for a size distribution of grains support this conclusion (Hanner 1983; Hanner et al. 1985). The comets with the strongest dust emission, such as Halley, have higher color temperature at $3.5-8 \mu \mathrm{m}$ than at $8-20 \mu \mathrm{m}$, indicating an enhanced abundance of hot, sub- $\mu \mathrm{m}$ grains. Silicates must be sufficiently mixed with absorbing material that they are warm and dark. Only a small imaginary component in the refractive index, $n^{\prime \prime} \gtrsim 0.01$ is actually needed to raise the temperature of micron-sized silicate grains (Hanner 1983); this could be due to finely dispersed carbonaceous material or to the FeNi beads found within the glassy silicate particles described by Bradley (1995).

While the $3-20 \mu \mathrm{m}$ thermal emission allows us to determine the amount of small dust in the coma, these data do not help us determine the amount of mass in large particles, with their lower ratio of cross section to mass. The dust impact detector (DIDSY) on GIOTTO recorded a very broad mass distribution (McDonnell et al. 1991). The small slope at masses $>10^{-9} \mathrm{~kg}$ implied that the mass was concentrated in these large particles. Meteor streams associated with comets contain particles in this size range. Radar observations of comets IRAS-Araki-Alcock (Goldstein et al. 1984; Harmon et al. 
1989) and P/Halley (Campbell et al. 1989) detected the presence of large grains surrounding the nucleus.

While these particles are "invisible" in the infrared, their thermal radiation may be detectable at longer wavelength, since the emissivity of the small grains will decrease as $\lambda^{-\alpha}, 1 \leq \alpha \leq 2$, while the emissivity of the large grains remains essentially constant. Jewitt and Luu (1990) detected emission from P/Brorsen-Metcalf at $800 \mu \mathrm{m}$. If one compares the flux with the $10 \mu \mathrm{m}$ flux recorded 4 days earlier, one finds that the data are consistent with a radiating blackbody having constant emissivity (Lynch et al. 1992a). That is, the large grains emitting at $800 \mu \mathrm{m}$ can account for the $10 \mu \mathrm{m}$ flux as well; Brorsen-Metcalf apparently lacked the population of small grains present in most comets, consistent also with the lack of a silicate feature and the low scattered light continuum. In contrast, four other comets measured by Jewitt and Luu (1992) have submillimeter fluxes or upper limits that are one to two orders of magnitude lower than the flux extrapolated from $10 \mu \mathrm{m}$ on the assumption of constant emissivity

\section{Infrared Spectral Features}

\section{Hydrocarbons}

A broad emission feature centered near $3.36 \mu \mathrm{m}$ was discovered in the Vega IKS spectra of Halley (Combes et al. 1988). The emission is attributed to the stretching vibration of $\mathrm{C}-\mathrm{H}$ bonds in organic molecules. Subsequent spectra of Halley from ground-based telescopes revealed spectral structure (Fig. 1); the feature persisted to at least $2 \mathrm{AU}$ (Baas et al. 1986). A similar emission feature has been detected in every bright comet since Halley, including new, long-period and periodic comets. The strength of the feature correlates with the water production rate, rather than with the continuum brightness, suggesting that the carrier is most likely a gas phase species (Brooke et al. 1991).

The narrower emission feature at $3.52 \mu \mathrm{m}$ has been attributed to the $v^{3}$ band of methanol, based on the good agreement between the abundance of methanol derived from fitting the infrared feature and that derived from detection of methanol at millimeter wavelengths (Hoban et al. 1991). Methanol has other vibrational bands at $3.33 \mu \mathrm{m}\left(\nu^{2}\right)$ and $3.37 \mu \mathrm{m}\left(\nu^{9}\right)$ and these will contribute to the broad $3.36 \mu \mathrm{m}$ emission (Reuter 1992). Careful modeling of the methanol bands leads to a residual feature centered at 3.424-3.43 $\mu \mathrm{m}$ (Bockelée-Morvan et al. 1995; DiSanti et al. 1995). The strength of the residual feature correlates with water production rates and especially with the methanol abundance; thus, it is likely to arise from a gaseous species. Assuming a $\mathrm{g}$-factor comparable to that of methanol, the abundance of the carrier is comparable to methanol, $\sim 4 \%$ relative to water, making it a significant reservoir of carbon. Overtone and combination bands of methanol may also contribute. Further progress will require high spectral resolution to resolve line structure and sort out the methanol contribution.

A small, but distinct, feature at $3.29 \mu \mathrm{m}$ is present in the spectra of the dusty comets Halley (Fig. 1), Levy 1990 XX (Davies et al. 1991), and Swift-Tuttle (Di Santi 


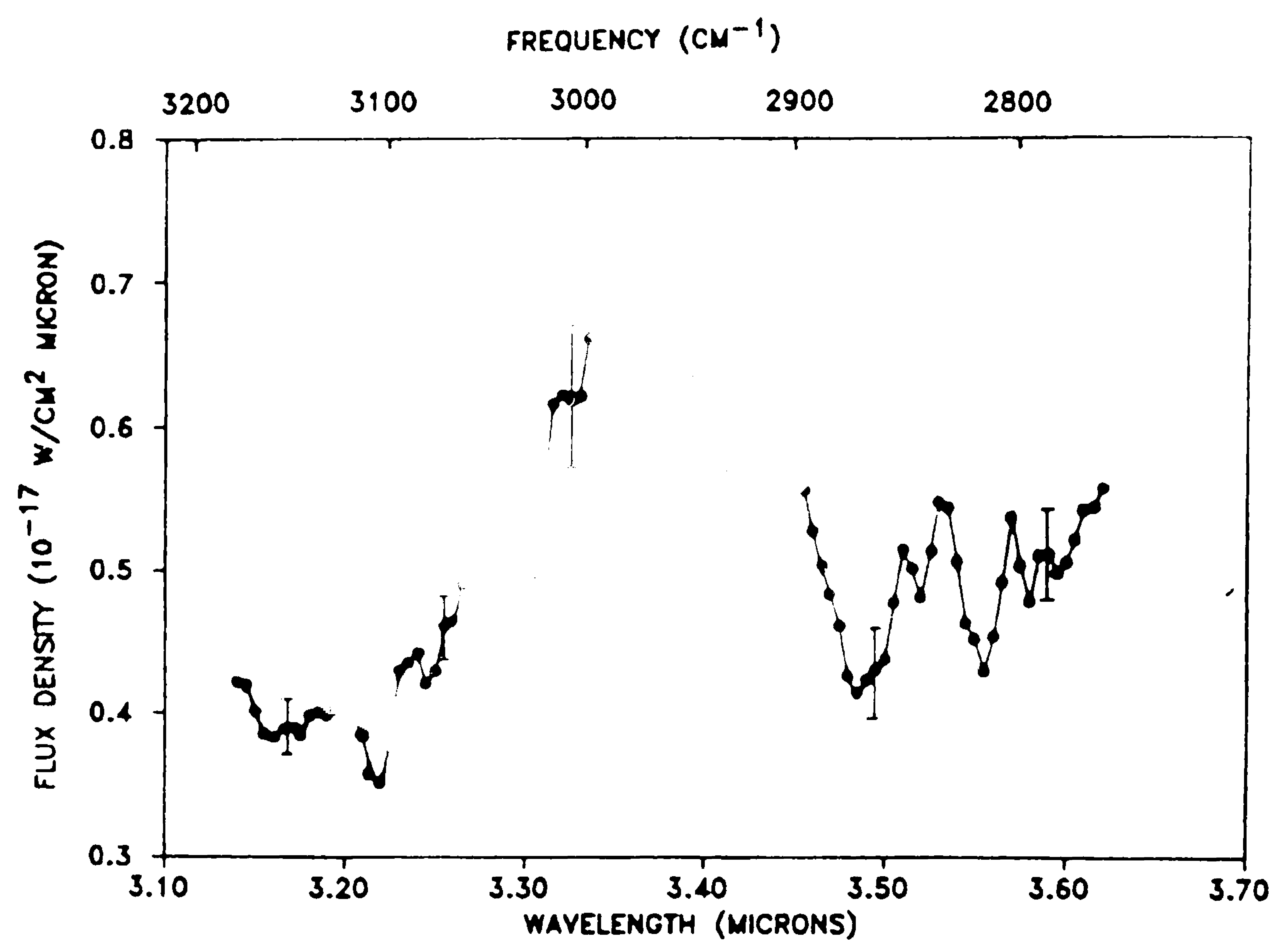

Fig. 1. Spectrum of P/Halley 25 April 1986 at $\mathrm{R}=1.56 \mathrm{AU}$ (Baas et al. 1986)

et al. 1995). In Swift-Tuttle, the feature appeared to be stronger on Nov 27, when the dust continuum was also stronger. Typical of aromatic bonds, this feature could arise from polycyclic aromatic hydrocarbons either in molecules or small solid grains. An interstellar feature at $3.29 \mu \mathrm{m}$ is associated with other bands at $6.2,7.7,8.6$, and 11.3 $\mu \mathrm{m}$. No evidence of the 6.2 and $77 \mu \mathrm{m}$ bands is seen in the only $5-8 \mu \mathrm{m}$ spectra of comets Halley (Bregman et al. 1987) and Wilson 1987 VII (Lynch et al. 1989).

Thus, to date, there is no positive identification of a spectral feature from $\mathrm{CHON}$ grains, but further study of the $3.29 \mu \mathrm{m}$ feature is warranted. Indeed, PAHs have been discovered in IDPs (Clemett et al. 1993).

\section{Silicates}

A silicate emission feature near $10 \mu \mathrm{m}$ is seen in a number of comets. The shape of the feature can be diagnostic of the silicate mineralogy (e.g. Hanner et al. 1994a). Four comets (among the 9 with good spectra) display a strong feature with a peak at $11.2 \mu \mathrm{m}$ arising from crystalline olivine (Fig. 2). These are long-period comets Bradfield 1987 XXIX (Hanner et al.1990) and Levy 1990 XX (Lynch et al. 1992b), new comet Mueller 1993a (Hanner et al. 1994b) and P/Halley (Bregman et al. 1987; Campins \& Ryan 1989).

The olivine identification is based on the good spectral match with the measured spectral emissivity of Mg-rich olivine (Stephens \& Russell 1979) and the fact that the peak is seen only in association with a strong silicate feature. Olivine is present in chondritic aggregate IDPs. Other possible materials, such as $\mathrm{SiC}$ or hydrocarbons, can be ruled out from the width of the peak, abundance arguments, or for lack of the corresponding features, such as the $7.7 \mu \mathrm{m}$ and $8.6 \mu \mathrm{m}$ emission bands present with the $11.25 \mu \mathrm{m}$ PAH feature in interstellar sources. 


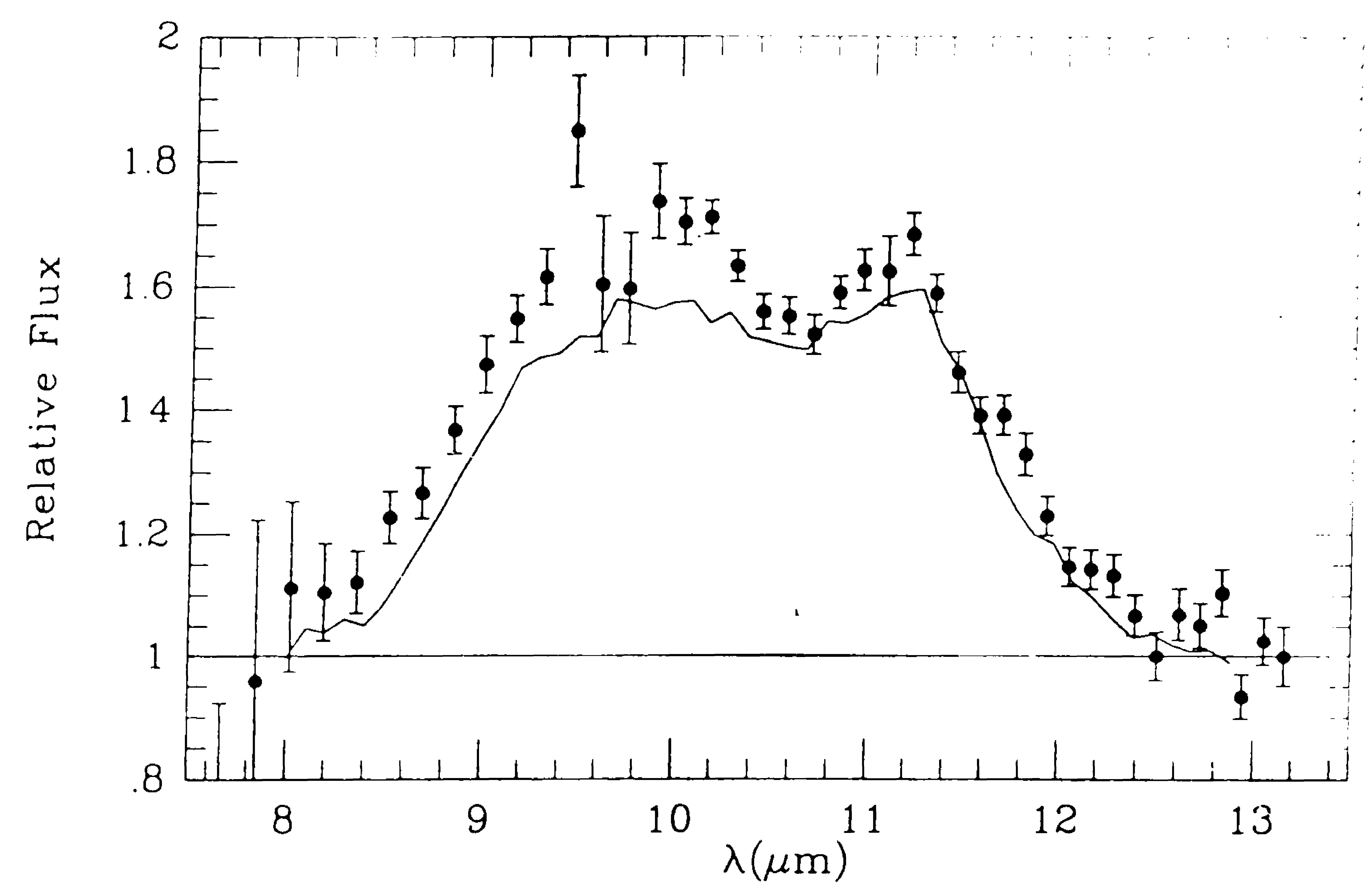

Fig. 2 Silicate feature in Mueller 1993a at $\mathrm{R}=2.06 \mathrm{AU}$ (Hanner et al. 1994b). Flux divided by $220 \mathrm{~K}$ blackbody continuum. Solid line is spectrum of $\mathrm{P} /$ Halley at $\mathrm{R}=0.79 \mathrm{AU}$ (Campins and Ryan 1989).

This result is significant because olivine is a high-temperature condensate. It can form by direct vapor condensation at $\mathrm{T} \sim 1400 \mathrm{~K}$ followed by slow cooling or by annealing of amorphous silicate grains at $\sim 900 \mathrm{~K}$. Such heating did not take place in the outer solar nebula. The spectral signature of olivine is rarely, if ever, seen in the interstellar medium. Thus, its presence in comets may be evidence of the transport of material from the warm inner solar nebula to the region of comet formation. But, so far, the $11.2 \mu \mathrm{m}$ peak has been seen in only a few comets. A wider sample of cometary spectra - and correlation with other dust properties - are needed to follow up on this potentially important subject.

The broad $9.8 \mu \mathrm{m}$ maximum in the comet spectra is similar to the interstellar feature and most likely arises from amorphous or glassy particles. Glassy silicate grains are common in chrondritic aggregate IDPs. Bradley $(1994,1995)$ argues that these are, in fact, interstellar grains with significant radiation damage before being incorporated into comets.

The spectra of four other new comets discussed in Hanner et al. (1994a) are puzzling; each has a unique, and not understood, spectrum. In Wilson $1987 \mathrm{VII}$, the feature is extremely broad, suggesting a very amorphous silicate material. We may be witnessing the effect of cosmic ray damage to the outermost layer of the nucleus over the lifetime of the Oort Cloud.

Other comets, such as P/Brorsen-Metcalf (Lynch et al. 1992a) and many short-period comets lack a silicate feature. The size of the dust grains affects the visibility of the silicate feature. These comets may lack a silicate feature because there is a deficiency of small grains rather than a lack of silicate material. 




Fig. 3 Mean albedo of comet grains versus phase angle at $\mathrm{J}(1.25 \mu \mathrm{m})$ (Hanner \& Newburn 1989).

\section{Optical Properties}

The optical properties of the dust provide information about the size and structure of the dust particles. While early interpretations were based on Mie theory, strictly valid only for smooth spheres, more recent work emphasizes scattering by irregular particles, as discussed at this Colloquium.

To determine the scattering phase function requires observations over an extended time period as the sun-comet-earth geometry changes. Consequently one has the problem of normalization to account for the changing dust production rate; typically, the gas production rate is used for normalizing the visual continuum, assuming a constant dust/gas ratio. Meech \& Jewitt (1987) analyzed the data for 4 comets observed at phase angle $0^{\circ}<\theta<25^{\circ}$. They find a linear slope of $0.02-0.035 \mathrm{mag} / \mathrm{deg}$. There was no evidence for an opposition surge larger than $20 \%$ in P/Halley at $1.37^{\circ}<\theta<8.6^{\circ}$ The phase function for the comets from $0^{\circ}-30^{\circ}$ is steeper than the volume scattering function of zodiacal dust derived by Lamy \& Perrin (1986), but less steep than that of dark asteroids. It is consistent with the phase function of fluffy absorbing particles (Hanner et al. 1981). Only two comets, West 1976 VI and Bradfield 1980t, have been observed at phase angle $150^{\circ}-120^{\circ}$ (Ney \& Merrill 1976; Ney 1982). The ratio of scattered to thermal energy shows strong forward scattering. The diffraction lobe appears to be wider for the comet dust than for the zodiacal light (Lamy 1985).

The geometric albedo of a particle, $A_{p}$, is defined as the ratio of the energy scattered at $0^{\circ}$ phase to that scattered by a white Lambert disk of the same geometric cross section (Hanner et al. 1981). Since comets are rarely observed at $0^{\circ}$ phase, it is convenient to define the quantity $A_{p}(\theta)$ at phase angle $\theta$, equal to the geometric albedo times the normalized phase function. 
Hanner \& Newburn (1989) summarized the $A_{p}(\theta)$ at $\mathrm{J}(1.25 \mu \mathrm{m})$ and $\mathrm{K}(2.2 \mu \mathrm{m})$ for several comets determined from simultaneous measurements of the scattered and thermal radiation. The albedos are very low, ranging from 0.025 at large phase angle to $0.05-0.10$ near $0^{\circ}$ phase in the $\mathrm{J}$ bandpass. Since distant comets tend to be the ones observed near $0^{\circ}$, a systematic bias may be present. Based on a comparison with the $A_{p}(\theta)$ derived for $\mathrm{P} /$ Halley at $13^{\circ}-18^{\circ}$, and with the shape of the phase function in the visible, it does appear that comets beyond $3 \mathrm{AU}$ have higher albedo, a conclusion also reached by Hartman et al. (1982), based on the near-infrared colors.

A higher albedo for the dust during times of strong jet activity was seen in comet Halley (Tokunaga et al. 1986). This could be due to a shift in the mean grain size or to a component of less-absorbing grains ejected during outburst. Laboratory measurements of the geometric albedo for irregular absorbing particles showed a size dependence from $A_{p} \sim 0.08$ for particle radius $2 \mu \mathrm{m}$ to $\sim 0.025$ for particle radius $60 \mu \mathrm{m}$ (Giese et al. 1986).

Albedo maps of comets $\mathrm{P} /$ Halley (Hammel et al. 1987), P/Giacobini-Zinner (Telesco et al. 1986), P/Brorsen-Metcalf (Ridgeway et al. 1991), and P/Swift-Tuttle (Fomenkova et al. 1994) were created by combining CCD images with thermal infrared images. The albedo is not constant across the coma, indicating variation in grain properties. In all 4 cases, the albedo increases radially from the nucleus. The lowest albedo occurs on the anti-sunward side of the nucleus in Halley and Giacobini-Zinner.

The color of the scattered light is generally redder than the sun; the reflectivity gradient decreases with wavelength from $5-18 \%$ per $0.1 \mu \mathrm{m}$ at $\lambda 0.35-0.65 \mu \mathrm{m}$ to $0-2 \%$ per $0.1 \mu \mathrm{m}$ at 1.6-2.2 $\mu \mathrm{m}$ (Jewitt \& Meech 1986). The dust is not necessarily the same color as the nucleus. In the case of $\mathrm{P} / \mathrm{Tempel} 2$ the $\mathrm{J}-\mathrm{H}$ color of the nucleus was up to 0.3 mag redder than that of the dust (Tokunaga et al. 1992).

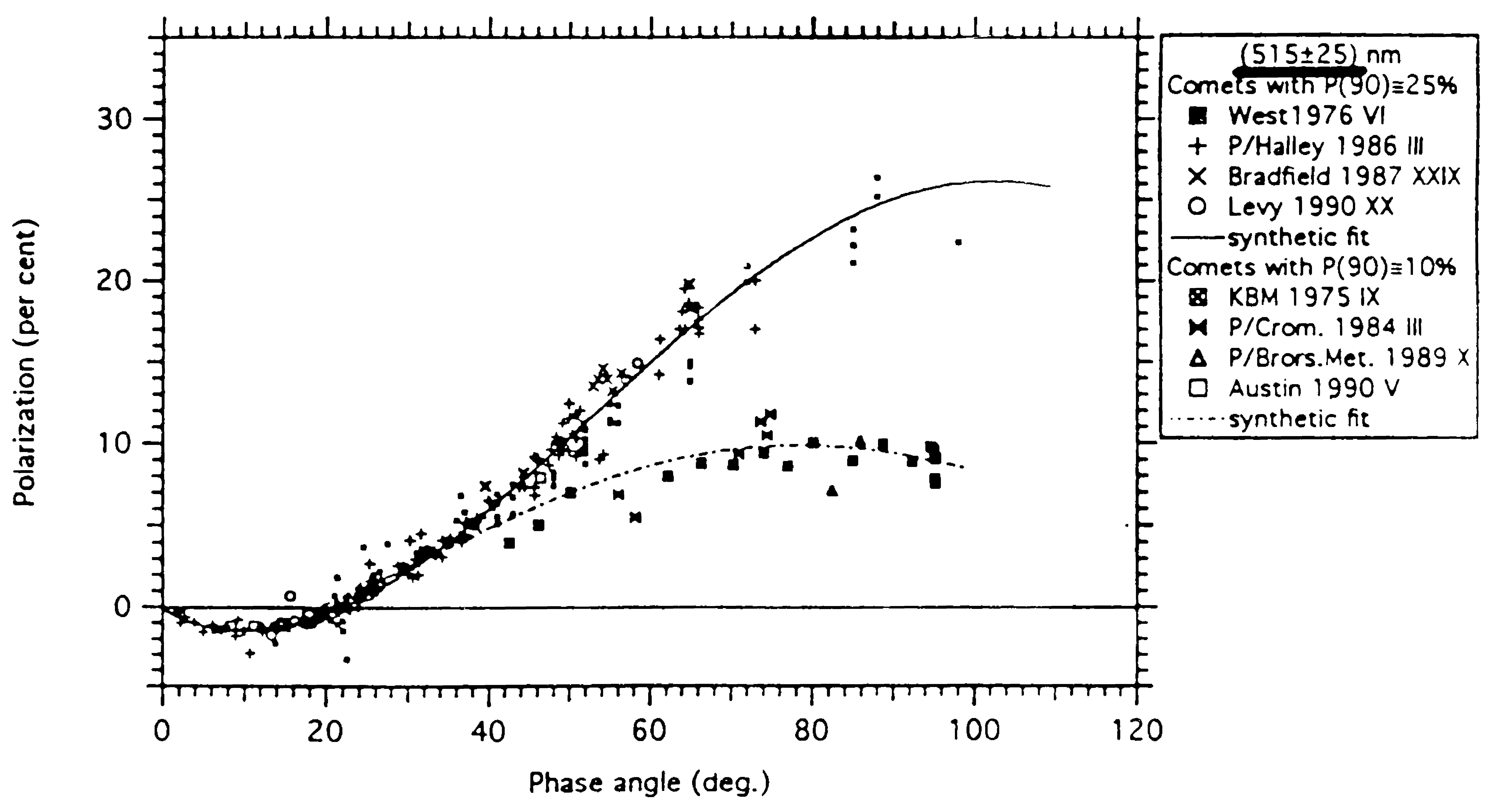

Fig. 4 Polarization versus phase angle at $\lambda=0.52 \mu \mathrm{m}$ (Levasseur-Regourd et al. 1995) 
The polarization phase curve has been measured for a number of comets (Oishi et al. 1978; Dollfus et al. 1988; Chernova et al. 1993; Levasseur- Regourd et al. 1995). Polarization has to be observed through filters that isolate the continuum; some earlier measurements were contaminated by gas emission. All of the comets show negative polarization at small phase angles, a neutral point at $\theta_{c}=21 \pm 2^{\circ}$, slope $h \sim 0.2-0.3 \% / \mathrm{deg}$ at $\theta_{c}$, and maximum polarization near $90^{\circ}$. However, they separate into two groups having $P_{\max } \sim 10-15 \%$ and $\sim 25 \%$ respectively. The higher $P_{\max }$ correlates with a stronger dust continuum and stronger silicate emission feature. For sub- $\mu \mathrm{m}$ to $\mu \mathrm{m}$ particle sizes, $P_{\max }$ decreases with increasing particle size, as do albedo and silicate emission. Thus, the two groups of comets may have differences in their dust size distribution, although differences in the composition (presence of small "clean" silicates?) or structure (fluffy aggregates?) of the grains could also contribute. In the case of Halley, the polarization at large phase angles increased with wavelength (Dollfus et al. 1988), as one would expect if particle size is controlling $P_{\max }$.

Spatially resolved observations of the inner coma often show increased polarization in jets (Eaton et al. 1988). In their study of polarization in $\mathrm{P} / \mathrm{Halley}$, Dollfus et al. (1988) distinguish 3 regions: a bright inner halo (radius $r \sim 100 \mathrm{~km}$ ), the inner coma or "fresh" dust $(r<5,000 \mathrm{~km})$ and the outer coma $(r \sim 10,000 \mathrm{~km})$. The inner coma consistently produced higher polarization at large phase angles, while the halo displayed lower polarization than the other regions at $\theta=30-40^{\circ}$. Lower albedo near the nucleus was also apparent in Halley; both features could be consistent with an excess of larger particles surrounding the nucleus.

\section{Future Directions}

Continuing improvements in ground-based and space-based instrumentation are creating new opportunities for a better understanding of cometary dust.

The sub-arcsec spatial resolution now possible with modern optical and infrared arrays at large ground-based telescopes will allow us to trace the spatial distribution of particles of different size and composition, based on their thermal emission, albedo, color, and polarization. Coordinated infrared, optical, and polarization maps are needed to accomplish this goal. The lower albedo and polarization observed in the near-nucleus region of the coma are not yet satisfactorily explained. Along with these observations, more work on the scattering properties of irregular particles is needed, particularly to understand the polarization.

High resolution $3 \mu \mathrm{m}$ spectra are required to solve the mystery of the $3.36 \mu \mathrm{m}$ $\mathrm{C}-\mathrm{H}$ emission feature and learn more about the $3.29 \mu \mathrm{m}$ feature. Spectroscopy of comets beyond $3 \mathrm{AU}$ may detect icy grains, particularly the $3 \mu \mathrm{m}$ water ice feature, as well as volatile organic species.

The origin of the crystalline olivine grains remains a puzzle. So far, the $11.2 \mu \mathrm{m}$ olivine feature has been seen in only 4 comets and we need to understand why it appears in some comets and not others.

More sensitive submillimeter detectors will allow detection of thermal emission, 
or useful upper limits, to constrain the abundance of large particles in the coma. This is important for determining the total rate of mass loss from the nucleus.

The ISO satellite will make infrared photometric, spectroscopic, and imaging observations of several comets in the next 18 months. The entire infrared spectrum from $3-200 \mu \mathrm{m}$ will be accessible. We can expect new insights from these observations.

Acknowledgements. This research was performed at the Jet Propulsion Laboratory, California Institute of Technology, under contract with the National Aeronautics and Space Administration.

\section{References}

Baas, F., Geballe, T R. and Walther, D. M. 1986, ApJ Lett. 311, L97

Bockelée,-Morvan, D., Brooke, T Y and Crovisier, J. 1995, Icarus 116, 18

Bradley, J. P. 1994, Science 265, 925

Bradley, J. P 1995, this volume

Bregman, J. et al. 1987, A\&A 187, 616

Brooke, T Y., Tokunaga, A. T and Knacke, R. F. 1991, AJ 101, 268

Brownlee, D. 1995, these proceedings

Campbell, D. B., Harmon, J. K. and Shapiro, I. I. 1989, ApJ 338, 1094

Campins, H. and Ryan, E. 1989, ApJ 341, 1059

Chernova, G. P., Kiselev, N. N. and Jockers, K. 1993, Icarus 103, 144

Clemett, S. J., Maechling, C. R., Zare, R. N., Swan, P. D. and Walker, R. M. 1993, Science 262, 721

Combes, M. et al. 1988, Icarus 76, 404

Davies, J. K., Green, S. F and Geballe, T R. 1991, MNRAS 251, 148

Di Santi, M. A., Mumma, M. J., Geballe, T. R. and Davies, J. K. 1995, Icarus 116, 18

Dollfus, A. Bastien, P., Le Borgne, J.-F., Levasseur-Regourd, A. C. and Mukai, T 1988, A\&A 206, 348

Duncan, M., Quinn, T and Tremaine, S. 1988, ApJ Lett. 328, L69

Eaton, N., Scarrott, S. M. and Warren-Smith, R. F. 1988, Icarus 76, 270

Fomenkova, M., Larson, S., Jones, B. and Pina, R. 1994, Bull. AAS 26, 1119

Giese, R. H., Killinger, R. T., Kneissel, B. and Zerull, R. H. 1986, ESA SP-250, II, p. 53

Goldstein, R. M., Jurgens, R. F. and Sekanina, Z. 1984, AJ 89, 1745

Hammel, H. B., Telesco, C. M., Campins, H., Decher, R. Storrs, A. D. and Cruikshank, D P. 1987, A\&A 187, 665

Hanner, M. S. 1983, in Cometary Exploration, ed. T I. Gombosi, II, 1

Hanner, M. S. et al. 1985, Icarus 64, 11

Hanner, M. S. and Newburn, R. L. 1989, AJ 97, 254

Hanner, M. S., Giese, R. H., Weiss, K. and Zerull, R. 1981, A\&A 104, 42

Hanner, M. S., Hackwell, J. A., Russell, R. W and Lynch, D. K. 1994b, Icarus 112, 490 Hanner, M. S., Lynch, D. K. and Russell, R. W. 1994a, ApJ 425, 274

Hanner M. S., Newburn, R. L., Gehrz, R. D., Harrison, T., Ney, E. P and Hayward, T 
L. 1990, ApJ 348, 312

Harmon, J. K., Campbell, D. B., Hine, A. A., Shapiro, I. I. and Marsden, B. G. 1989, ApJ 338,1071

Hartmann, W K., Cruikshank, D. P., and Degewij, J. 1982, Icarus 52, 377

Hoban, S., Mumma, M. J., Reuter, D. C., Di Santi, M. A., Joyce, R. R. and Storrs, A. 1991, Icarus 93, 122

Jessberger, E. K., Christoforidis, A. and Kisssel, J. 1988, Nature 332, 691

Jewitt, D. C. and Luu, J. X. 1995, AJ 109, 1867

Jewitt, D. C. and Luu, J. X. 1992, Icarus 100, 187

Jewitt, D. C. and Luu, J. X. 1990, ApJ 365, 738

Jewitt, D. C. and Meech, K. J. 1986, ApJ 310, 937

Kissel, J. et al. 1986, Nature, 321, 280, and 336

Lamy, Ph. L. 1985, in Asteroids, Comets, Meteors II, ed. Lagerkvist et al. 373

Lamy, Ph. L. and Perrin, J.-M. 1986, A\&A 163, 269

Levasseur-Regourd, A. C., Dumont, R., Goidet-Devel, B., Hadamcik, E., Renard, J. B. 1995 , in preparation.

Lynch, D. K. et al. 1989, Icarus 82, 379

Lynch, D. K., Hanner, M. S., and Russell, R. W. 1992a, Icarus 97, 269

Lynch, D. K., Russell, R. W., Hackwell, J. A., Hanner, M. S. and Hammel, H. B., 1992b, Icarus 100,197

Meech, K. J. and Jewitt, D. C. 1987, A\&A 187, 585

McDonnell, J.A.M., Lamy, P. L. and Pankiewicz, G. S. 1991, in Comets in the PostHalley Era, ed. R. L. Newburn, M. Neugebauer, and J. Rahe, Kluwer Dordrecht, 1043.

Ney, E. P 1982, in Comets, ed. L. L. Wilkening, p. 323

Ney, E. P and Merrill, K. M. 1976, Science 194, 1051

Oishi, M., Kawara, K., Kobayashi, Y., Maihara, T. Noguchi, K., Okuda, H. and Sato, S. 1978, Pub. Astron. Soc. Japan 30, 149

Ridgeway, S. E. Jewitt, D., Campins, H., Luu, J., Joy, M., Sisk, C., and Telesco, C. M. 1991, in Astrophysics with Infrared Arrays, ASP Conf. Ser. 14, 329

Reuter, D. C. 1992, ApJ 386, 330

Stephens, J. R. and Russell, R. W 1979, ApJ 228, 780

Telesco, C. M. et al. 1986, ApJ. Lett. 310, L61

Tokunaga, A. T., Golisch, W. F., Griep, D. M., Kaminski, C. D. and Hanner, M. S. 1986, AJ 92, 1183

Tokunaga, A. T., Hanner, M. S., Golisch, W. F., Griep, D. M., Kaminski, C. D. and Chen, H. 1992, AJ 104, 1611

Xing, Z. and Hanner, M. S. 1995, this volume 